\title{
A single-center, prospective, randomized clinical trial to study the effectiveness of exercise therapy in patients with Cl-type Osteonecrosis of the femoral head: study protocol for a randomized controlled trial
}

Zhi ming Wu

Guangzhou University of Chinese Medicine

Ming jia Zhang

Guangzhou University of Chinese Medicine

Hai cheng Chen

Guangzhou University of Traditional Chinese Medicine First Affiliated Hospital

\section{Zhao jun Yang}

Guangzhou University of Chinese Medicine

\section{Peng Wu}

Guangzhou University of Chinese Medicine

Zhi jun Yue

Guangzhou University of Chinese Medicine

Duo rui Nie

Guangzhou University of Chinese Medicine

Chi Zhou (D642899538@qq.com)

Guangzhou University of Traditional Chinese Medicine First Affiliated Hospital

\section{Study protocol}

Keywords: Osteonecrosis of the femoral head, shock wave, exercise treatment, Tongluo Shenggu Jiaonang

Posted Date: August 12th, 2019

DOI: https://doi.org/10.21203/rs.2.12624/v1

License: (c) (1) This work is licensed under a Creative Commons Attribution 4.0 International License.

Read Full License 


\section{Abstract}

[EXSCINDED] Background Appropriate exercise training is beneficial to improve the clinical efficacy and early recovery of patients with Osteonecrosis of the femoral head. There is currently no effective lifestyle recommendation for patients with Osteonecrosis of the femoral head and lack of data demonstrating the clinical efficacy of exercise therapy for patients with Osteonecrosis of the femoral head. Our study is aimed to determine the effectiveness of exercise in the treatment of Cl-type Osteonecrosis of the femoral head.Design 866 participants who met the inclusion criteria will be randomly assigned to the experimental or control group. The experimental group will carry out a two-year shock wave treatment with exercise training and oral Tongluo Shenggu Jiaonang treatment, the control group will only carry out a two-year shock wave treatment with oral Tongluo Shenggu Jiaonang intervention. The clinical efficacy and imaging changes between the two groups will be compared to determine if exercise training is helpful for Osteonecrosis of the femoral head.Discussion This is a prospective, single-center, randomized controlled trial to determine if exercise training is effective for Osteonecrosis of the femoral head.

\section{Background}

Collapse is the turning point in the treatment of Osteonecrosis of the femoral head, the result of the combined effect of repair reaction and stress, and has a direct relationship with biomechanical factors. Stress concentration around the necrotic bone plays an important part role in the collapse of the necrotic femoral head. In normal gait, hip joint needs to bear $30 \%$ of the human body weight, which not only affects the blood supply in the femoral head, but also increases the bone marrow pressure in the proximal femur and causes pain..$^{1]}$ Therefore, non-weight-bearing treatment, as a non-surgical physical intervention, was first proposed for early ONFH treatment, through changes in activities such as bed rest, wheelchairs, walking aids, etc. or surgery to change the state of the femoral head, such as rotary osteotomy. ${ }^{[2]}$

However, Mont et al. in two articles found that wheelchairs or walkers cannot achieve satisfactory clinical results in reducing patient weight bearing through systematic evaluation ${ }^{[3,4]} .59 \%$ of patients (394 of 664 hips) developed pain during the average follow-up of 7 years or the Osteonecrosis of the femoral head developed into a collapsed period. Okazaki et al. found that there was no significant difference in the progression of ONFH between negative and non-negative recombination, so the authors believed that weight-bearing did not lead to disease progression in non-traumatic ONFH in rats ${ }^{[5]}$.

Exercise therapy refers to the use of instruments, freehand or the patient's own strength, through certain movements (active or passive exercise, etc.), standardized training of the muscles around the patient's diseased joints, in order to relieve joint pain, inhibit pathological development, improve muscle atrophy and muscle strength, increase joint stability and improves joint function. Clinically, the effect of exercise therapy on knee osteoarthritis has been confirmed by a large number of literature and cases ${ }^{[6,7]}$. The activity of the knee joint not only promotes the metabolism of cartilage, but also provides nutrients for the

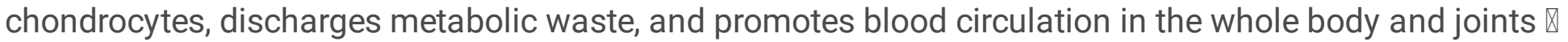


which is inextricably linked to the causes of osteonecrosis of the Osteonecrosis of the femoral head, blood circulation interruption and other causes. Unlike combined core decompression, fenestration, drilling or other surgical interventions having been confirmed, or shock wave, high pressure oxygen, pulsed electromagnetic field and other treatments used as the main treatment of $\mathrm{ONFH}^{[8,9]}$, exercise therapy is rare at home and abroad that restore the muscle strength and response of the muscles around the hip to normal or near normal levels through specific weight loss training, Strengthen the stability of the core muscles of the waist and abdomen, coordinate the dynamic balance between the adductor muscles and the abductor muscles, maximize the function of necrotic femoral head, and alleviate the clinical symptoms of patients.

In essence, ONFH is the damage or interruption of blood supply in the femoral head, causing the death and repair of bone cells, secondary micro-fractures, subchondral fractures, "stress concentration", "intra head instability", resulting in femoral articular surface collapse, osteoarthritis ${ }^{[10]}$. The "collapsed collapse period" includes the early collapse and collapse of the early stage. The JIC CI type is equivalent to the early collapse stage. According to Wolff law, the anterior and lateral part of the femoral head is the main way of stress conduction in the femoral head and neck, and is the main mechanical support of the femoral head. The early stage is the best time to protect the hip. It can also be understood as the best intervention period to avoid the collapse of the femoral head joint surface and form hip osteoarthritis.

Therefore, we have reason to believe that the movement of the waist and abdomen muscles can greatly relieve the compression stress of the femoral head, delay the collapse of the articular surface of the femoral head and the occurrence of hip osteoarthritis, and delay the time of joint replacement.

\section{Design}

This study is a non-inferiority trial and is designed for a prospective, single-center, randomized, parallelgroup, single-blind, clinical controlled study. Patients with Osteonecrosis of the femoral head will be recruited and divided into two groups: the exercise treatment group (intervention group) and the conventional treatment group (control group).

The sample size is estimated as follows. According to the results of related literature, it is suggested that the experimental group is expected to have an effective rate of $86 \%$, and the control group is expected to have an effective therapeutic rate of $79 \%$. This study is expected to be recruited for 2 years, with a total observation time of 5 years, using the $Z$ test, $a=0.15, \beta=0.1$ (power $=90 \%$ ). We will also use PASS 16 software Tests for Two Proportions to estimate. There are no fewer than 433 eligible subjects in each group ${ }^{[11-13]}$.

\section{Outcomes}

\section{Primary outcomes}




\section{-VAS Pain Score}

The subjects will be compared with the hip joint pain related indicators of visual analogue scale (VAS) every three months to observe the changes of hip joint pain in the two groups.

\section{-Harris Hip Score}

The Harris hip score will be used to assess the efficacy of exercise therapy with a total score of 100 . Higher score indicates better functional recovery. Harris score at the end of 0, 3 months, 6 months, 9 months, 12 months, 15 months, 18 months, 21 months, and 24 months after surgery will be used.

\section{-WOMAC Score}

WOMAC hip score will be used to assess the efficacy of exercise therapy. VAS will be utilized to evaluate each questionnaire question. The total index score will be expressed by the total number of points in 24 components. The higher the WOMAC index, the more intense the OA and the worse the functional recovery. Harris score in this study is quantitative, and the WOMAC score at the end of 0, 3, 6 months, 9 months, 12 months, 15 months, 18 months, 21 months, and 24 months after surgery will be used ${ }^{[14]}$.

\section{-Hip Joint Radiograph}

The ARCO staging, JIC typing, and frog position typing will be performed on the imaging data, and the aspect ratio, lateral edge height, sharp angle, CE angle, CME angle, Tony angle, and head lice index will be measured.

\section{Secondary outcomes}

\section{-Hip pain time}

\section{-Quality of life assessment}

\section{Participation center and investigator}

This will be a single-center study, with all patients from the First Affiliated Hospital of Guangzhou University of Traditional Chinese Medicine. Doctors and rehabilitators participating in the study received 
over 500 patients with Osteonecrosis of the femoral head each year. Each researcher will participate in training of the treatment of Osteonecrosis of the femoral head.

\section{Registration}

The trial protocol has been approved by the Ethics Committee of the First Affiliated Hospital of Guangzhou University of Traditional Chinese Medicine (ZS-1269) and has been registered at chictr.org (identification number ChiCTR1900023963). All eligible participants and/or their legal representatives will be fully aware of the potential risks and benefits of each group of interventions. Only patients who provide written informed consent will participate in the trial.

\section{Participant}

Study participants will be patients with Osteonecrosis of the femoral head JIC type Cl type collapse.

\section{Criteria}

\section{Eligibility criteria}

-Age 18 to 50

-Complies with the diagnostic criteria for the JIC classification of the Osteonecrosis of the femoral head

\section{Exclusion criteria}

-Age less than 18 or older than 80

-Does not meet the diagnostic criteria for the JIC classification of the Osteonecrosis of the femoral head

-Previous femoral head surgery

-People who cannot complete treatment for 24 consecutive months

\section{Exit criteria}

In any visit, after complete and standard diagnostic procedure, patients with hip pain time more than 6 months and a femoral head collapsed $>4 \mathrm{~mm}$ X-ray film result, will be identified as completed cases and study termination. The entire content of visit 50 (V50) must be accomplished before withdrew from the trial (see the "Research Flow Chart" for details).

\section{Randomization And Blinding}


The order of assignment is generated by random numbers, subjects will be selected by an outpatient specialist, and subjects will be assigned to each intervention group by a third party independent of the trial practitioner and the clinical therapist. The evaluator will be blinded by the specificity of the treatment, and the evaluator will be performed by an independent researcher who do not know the exercise treatment procedure.

\section{Definitions}

Test group: The subject received shock wave treatment, exercise training and took Tongluo Shenggu capsules orally. The subject was placed in the supine position. On the first day, the position of the femoral head was accurately located through the external anatomical landmark around the ill side hip. The shock wave high energy head was selected, the intensity was 2.0 bars, and the femoral head was continuously impacted 2000 times. The next day, the lower part was selected. The energy head is $1.0 \mathrm{bar}$, and the soft tissue around the hip joint is continuously impacted 2000 times. The plan was used alternately for 8 times. The subject was given the second shock wave treatment three months later and the third shock wave treatment after six months. After the shock wave treatment, the subject received sport training guidance and performed exercise training every day. Each exercise included eleven groups of hip joint training, six groups of core abdominal muscle exercises and ten groups of pelvic training. Each set of training was repeated 5-15 times from a given exercise of relatively low intensity, maintaining the same training intensity for two weeks. Resistance will increase when a subject is able to tolerate a given load with a comfortable load of 5-15 repetitions of a given exercise.

Control group: The subject received shock wave treatment and took Tongluo Shenggu capsule orally. The subject was placed in the supine position. On the first day, the position of the femoral head was accurately located through the external anatomical landmark around the ill side hip. The shock wave high energy head was selected, the intensity was 2.0 bars, and the femoral head was continuously impacted 2000 times. The next day, the lower part was selected. The energy head is $1.0 \mathrm{bar}$, and the soft tissue around the hip joint is continuously impacted 2000 times. This was used alternately for 8 times. The subject was given the second shock wave treatment three months later and the third shock wave treatment after six months ${ }^{[15]}$.

\section{Detailed Description Of The Program}

The initial screening assessment would be conducted 2 weeks prior to the start of the trial. A wrote informed consent form was given to the patient prior to screening. The lead-in period was 2 weeks, -2 weeks to 0 days. The work steps were as follows: Subjects sign informed consent; obtain demographic data, medical history and treatment history, drinking history; general physical examination (physique, body temperature, heart rate, respiratory rate, blood pressure, height, weight, BMI); give subjects exercise instruction materials and self-assessments; recycle form and perform hip joint frog position X-ray film; review the selection and exclusion criteria; perform the gestational pregnancy test for women of childbearing age; check the urine routine, blood routine (including C-reactive protein), blood uric acid, liver 
function (ALT, AST, TBIL, ALP, GGT), renal function (BUN, Scr, urinary microfilming, urinary N-kinase), and 12-lead ECG.

The day before the start of the trial, was at the baseline, the 0th day of the start of the trial. First the subject received general physical examination (heart rate, respiratory rate, blood pressure, height, weight, $\mathrm{BMI}$ ). Then we conducted a series of evaluations on patients, including Haris, VAS, WOMAC score, quality of life assessment; given the subject exercise instructional materials and self-evaluation form; perform hip joint frog position X-ray film; evaluated the clinical outcome of the patient and reviewed the inclusion/exclusion criteria. Next we conducted exercise therapy guidance for the selected subjects, recovered and distributed the self-evaluation form for the publicity and education compliance, obtained the random number, solicited the patient's will, conducted an intentional analysis, and randomly assigned the subjects to the test group and control group. The test group and control group were recorded for recent combined medication, alcohol consumption, and adverse events. After that, visits were conducted every two weeks, and every two weeks, according to the quality of the exercise performed by the patient, whether to upgrade to the next course of treatment. Each visit is conducted with general physical examination and exercise therapy guidance, and a self-evaluation form for the publicity and education compliance was issued. Record the recent combined medication, alcohol consumption, whether they are endpoint and adverse events. If an endpoint event occurs, we would complete an interview with all content and study it as a completed case. The progress of necrotic femoral head lesions was observed in hip joint frog position X-ray film every three months. See Table 2 for the schedule of clinical visits and clinical trial procedures.

\section{Exercise therapy training program:}

1. Hip abduction: supine position, horizontal abduction unilateral hip joint from 0 to 30 degrees, a group of 15 times, each time maintaining 30 degrees for 5 seconds, 4 groups a day, a group of 15 times.

2. Hip extension: prone position, unilateral extension of the hip joint 15 degrees, a group of 10 times each time to maintain the hip extension for 5 seconds, 4 groups a day, a group of 10 times.

3. Core abdominal muscle movement: supine position, with a sphere or column placed under the armpit, abdominal muscles and back muscles, body straight, shoulders, pelvis, knees in the same line, maintain 10 seconds, 6 groups a day, a group of 5 times.

4. Anterior and posterior movement of the pelvis: with the ischial tuberosity as the fulcrum, lift one side of the pelvis, coordinate the psoas and pelvic muscles, separate the hip muscles for movement, move the other side to the front, and then the other side of the ischial tuberosity The fulcrum, repeat the above action, the forward distance is 10 meters for a group, and 6 groups for 1 day.

5. pelvic coordination training: supine position, hands on the chest, with the ipsilateral upper ridge as the fulcrum, lifting the contralateral anterior superior ridge, a group of 15 times, each maintaining 30 degrees for 5 seconds, Practice four groups a day, a group of 15 times. 
6. Hip internal rotation: supine position, feet and shoulder width, ankle joint neutral position, hip joint as the center of rotation, feet swivel to the inside, internal rotation 10 degrees, 3 groups a day, a group of 10 times, each Maintain for 5 seconds $[12,16,17]$.

\section{Statistical Analysis}

\section{Case entry analysis}

List the total number of selected and completed cases and identify three analytical data sets (FAS, PPS, SS).

List cases of not entered PPS and their causes.

\section{Demographic data and baseline analysis}

Descriptive statistical demographic data and other baseline eigenvalues:

Continuous variables are computed for the number of cases, mean, standard deviation, median, minimum, and maximum.

Counting and grading data are utilized to calculate the frequency and composition ratio.

Inferential statistical results (P values) are listed as descriptive consequences.

\section{Efficacy analysis}

\section{Analysis of main efficacy indicators}

Clinical symptoms total score change rate was analyzed by variance analysis or rank sum test to compare differences between groups. The disappearance rate of the single symptom of clinical symptoms was compared with Chi-square test. The aspect ratio, sharp angle, CE angle, Tony angle, and head index change values were compared by $t$ test.

\section{Security analysis}

Calculated the incidence of adverse events and adverse reactions rate

According to different systems listed the frequency and frequency of adverse events and adverse reactions, and calculated the percentage 
Detailed list of cases of various adverse events

Detailed list of various adverse reactions

Laboratory indicators, electrocardiograms, and "normal abnormality" or "abnormal increase" number of cases and divergence rates of physical examinations after the test

Listed laboratory indicators, electrocardiograms, abnormal physical examination cases and clinical interpretations.

\section{Statistical software and general requirements}

Analysis using SPSS software.

All statistical tests used a two-sided test, and $\mathrm{P}$ value $\leq 5$ would be considered statistically significant.

Detailed statistical methods would be given in the statistical analysis plan.

\section{Interim analysis}

This study did not perform interim analysis.

\section{Discussion}

This experiment lacks effective monitoring of patients' exercise in non-hospital environments. Completion of recent exercise training is obtained from the patient's subjective perspective only based on the mission self-evaluation form. Lack of objective monitoring data. There is confined to the concealment of the patient's actual exercise effect. A fitness tracker can be issued on the subject to obtain objective data and supervise the subject, subject to research funding. Owing to the lack of blindness in this experiment, there may be selection bias. Because the experimental group and the control group were combined with oral Chinese patent medicine Tongluo Shenggu Jiaonang, there may be mixed bias.

\section{Trial Status}

Protocol version number: V2.0; protocol date: May 4, 2019, the date recruitment began: 2019/09/30, the approximate date when recruitment will be completed: 2020/2/28

\section{Abbreviations}

ONFH: Osteonecrosis of the femoral head; JIC: Japanese Investigation Committee; WOMAC: Western Ontario and McMaster Universities; BMI: Body Mass Index; ALT,AST: Aminotransferases; TBIL: total 
bilirubin; GGT: Gamma glutamyl transferase; RCT: Randomized controlled trial; CONSORT: Consolidated Standards of Reporting Trials; VAS: Visual Analogue Grading Method Score; SPSS: Statistical Product and Service Solutions; GCP: Good Clinical Practice; ALT: Alanine Transaminase; BUN: Blood Urea Nitrogen; Cr: Creatinine; Scr: Serum Creatinine; PASS: Power Analysis and Sample Size; SPIRIT: Standard Protocol Items: Recommendations for Interventional Trials; TCM: Traditional Chinese medicine;ECG: electrocardiogram; ITT: intention to treat; FAS: Full Analysis Set; PS: Per Protocol Set; SS: Safety Set; CE angle: Center-Edge angle;

\section{Declarations}

1. Acknowledgement

Acknowledgment to the rehabilitation therapists of the Department of Orthopaedics, the First Affiliated Hospital of Guangzhou University of Traditional Chinese Medicine.

\section{Funds}

This work is supported by National Natural Science Foundation of China (81873327) .The funders had no role in the design, execution, or writing of the study.

3. Availability of data and materials

Data sharing not applicable to this article as no datasets were generated or analyzed during the current study.

4. Author's contribution

ZM.W designed and developed the entire research program. MJ.Z participated in the writing of the manuscript. They are the main contributors to the manuscript. All authors read and endorsed the final draft.

5. Ethical approval and consent to participate

This trial has passed the ethical review of the Ethics Committee of the First Affiliated Hospital of Guangzhou University of Traditional Chinese Medicine. Ethical review approval number: NO.K [2019]024. Informed consent will be obtained from all study participants

\section{Consent for publication}

This manuscript doesn't contain any individual person's data in any form (including individual details, images or videos).

7. Conflict of interest

The authors declare that they have no competing interests.

\section{References}

[1] JIANG Delong and LI Keda, Osteonecrosis of the femoral head Laboratory Treatment and Observation Research Situation. Journal of Practical Traditional Chinese Internal Medicine, 2014. 28(5): p. 177-179. 
[2] HONG Zhi-nan et al., Current physiotherapy for osteonecrosis of the femoral head. Orthopedic Journal of China, 2017. 25(23): p. 2160-2164.

[3] Mont, M. A., J. J. Carbone and A. C. Fairbank, Core decompression versus nonoperative management for osteonecrosis of the hip. Clin Orthop Relat Res, 1996(324): p. 169-78.

[4] Mont, M. A., et al., The natural history of untreated asymptomatic osteonecrosis of the femoral head: a systematic literature review. J Bone Joint Surg Am, 2010. 92(12): p. 2165-70.

[5] Rantalainen, T., et al., Exercise loading does not account for polar distribution of cortical density at weight-bearing tibial mid-diaphysis. Osteoporosis International, 2011. 221: p. 50-51.

[6] Messier, S. P., et al., Exercise and dietary weight loss in overweight and obese older adults with knee osteoarthritis: the Arthritis, Diet, and Activity Promotion Trial. Arthritis Rheum, 2004. 50(5): p. 1501-10.

[7] Deyle, G. D., et al., Effectiveness of manual physical therapy and exercise in osteoarthritis of the knee. A randomized, controlled trial. Ann Intern Med, 2000. 132(3): p. 173-81.

[8] ZHU Heyu and ZHU Bing, Treatment of early stage avascular necrosis of the femoral head. China Journal of Orthopaedics and Traumatology, 2012. 25(7): p. 616-620.

[9] Gao, F., et al., High-Energy Extracorporeal Shock Wave for Early Stage Osteonecrosis of the Femoral Head: A Single-Center Case Series. Evid Based Complement Alternat Med, 2015. 2015: p. 468090.

[10] OU Zhixue, JIA Xiaojun and PANG Zhihui, Prof. He Wei's Experience in Treating Osteonecrosis of the femoral head and Collapse. Journal of New Chinese Medicine, 2011. 43(5): p. 155-156.

[11]C. Wang et al., "Extracorporeal shockwave therapy shows regeneration in hip necrosis," Rheumatology, vol. 47, no. 4, pp. 542, 2008.

[12]Liu Tiegang and Chen Weiheng, "Epidemiological study of non-traumatic Osteonecrosis of the femoral head," Contemporary Medicine, vol. 14, no. 17, pp. 2637-2639, 2009.

[13]Lv Benhao, "The Necrotic Location of Early Non-traumatic Osteonecrosis of The Femoral Head: A Clinical Investigation," Master degree, Zhengzhou University, 2018.,

[14]Chen Zhiwei et al., "Proposal on Bringing WOMAS and Holistic Health Evaluation Scale into Outcome Evaluation of Hip Protection Therapy for ANFH," World Chinese Medicine, no. 4, pp. 517-519, 2014.

[15] He Wei, "Scientific treatment of non-traumatic Osteonecrosis of the femoral head with traditional Chinese medicine," Chinese Journal of Joint Surgery(Electronic Edition), vol. 7, no. 3, pp. 284-286, 2013.

[16]Zeng Zhonghua et al., "Postoperative rehabilitation of patients with Osteonecrosis of the femoral head," Chinese Journal of Physical Medicine and Rehabilitation, vol. 27, no. 9, pp. 557-558, 2005. 
[17]Zhao Xiaohu, and Zhang Yong, "Literature Review to the Core Strength Exereises," Journal of Harbin Institute of Physical Education, vol. 29, no. 3, pp. 115-118, 2011.

\section{Tables}

Table 1:

\begin{tabular}{|l|l|}
\hline $\begin{array}{l}\text { Patients must meet all eligibility criteria and exclude all exclusion criteria before they can be included } \\
\text { in the study. } \\
\text { criteriaity }\end{array}$ & $\begin{array}{l}\text {-Age } 18 \text { to } 50 \text {-Complies with the diagnostic criteria for the JIC classification of the } \\
\text { femoral head necrosis }\end{array}$ \\
\hline $\begin{array}{l}\text { Exclusion } \\
\text { criteria }\end{array}$ & $\begin{array}{l}\text {-Age less than } 18 \text { or older than } 80 \\
\text {-Does not meet the diagnostic criteria for the JIC classification of the } \\
\text { femoral head necrosis } \\
\text {-Previous femoral head surgery } \\
\text {-People who cannot complete treatment for } 24 \text { consecutive months }\end{array}$ \\
\hline
\end{tabular}

Table 2: 


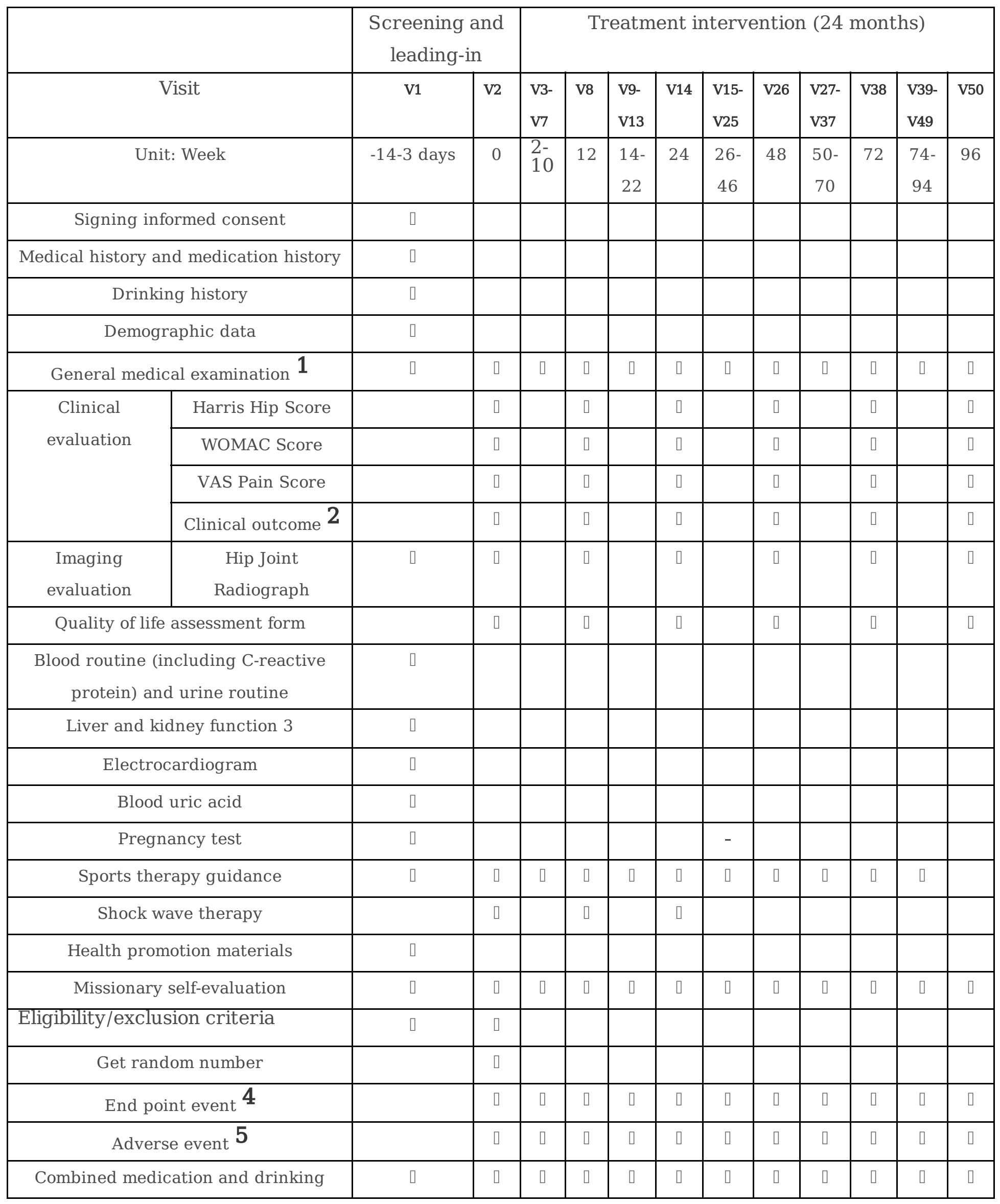


This is a list of supplementary files associated with this preprint. Click to download.

- renamed99775.pdf

- renamed9d086.pdf

- renamed9e8c7.jpg

- renamede5d94.pdf

- renamed5f4a8.rar

- renamed490b6.jpg

- renamed1eedb.rtf 